\title{
Weniger Symptome nach Training von Zungen- und Schluckmuskeln
}

Guimarães KC et al. Effects of oropharyngeal exercises on patients with moderate obstructive sleep apnea syndrome. Am J Respir Crit Care Med. 20o9;179:962-966

\section{Hintergrund}

Die Anwendung von kontinuierlichem, positivem Atemwegsdruck (CPAP) ist ohne Zweifel die effektivste Behandlung des obstruktiven Schlafapnoesyndroms (OSAS). Die Therapie ist allerdings gewöhnungsbedürftig, sodass Patienten mit mittlerem Schweregrad der OSA und geringer Symptomatik die Behandlung ablehnen oder nach alternativen Behandlungsmethoden suchen. Diese umfassen unter anderem die Anwendung von Unterkiefer-Protrusionsschienen, Gewichtsabnahme und operative Methoden wie die Uvulo-Palato-Pharyngoplastik.

\section{Fragestellung}

Es gab immer wieder Versuche, durch Training der oropharyngealen Muskulatur den Kollaps der oberen Atemwege im Schlaf zu verhindern. Deshalb entwickelte die Gruppe um Lorenzi-Filho ein Trainingsprogramm mit verschiedenen Übungen zur Stärkung der dilatierenden Muskulatur der oberen Atemwege.

\section{Methodik}

In einer randomisierten, kontrollierten Studie über drei Monate wurde ein Training der oropharyngealen Muskulatur durchgeführt. In die Kontrollgruppe wurden 15 Patienten, in die Behandlungsgruppe 16 Patienten einbezogen. Die Kontrollgruppe erhielt während einer wöchentlichen, überwachten Sitzung eine Scheintherapie mit Atemübungen durch die Nase, die täglich auch zu Hause während jeweils 30 Minuten durchgeführt werden sollte.

In der Studiengruppe wurden die Atemübungen durch ein Training der oropharyngealen Muskulatur ersetzt. Der weiche Gaumen wurde mit isotonischen und isometrischen Übungen trainiert. Dabei wurden der M. palatopharyngeus,
M. palatoglossus, M. uvulae, M. tensor veli palatini und der M. levator veli palatini einbezogen. Die Übungen bestanden außerdem aus einem Training der Zungen- und Gesichtsmuskulatur. Das Programm wurde durch ein Training der stomatognathischen Funktionen mit Atmen, Sprechen, Schlucken und Kauen ergänzt. Die jeweils drei Minuten dauernden Übungen summierten sich auf eine Trainingszeit von insgesamt 30 Minuten pro Tag.

\section{Ergebnisse}

Während in der Kontrollgruppe keine Änderungen auftraten, reduzierte sich die Tagesschläfrigkeit, gemessen mit der Epworth Sleepiness Scale (ESS), in der Gruppe mit oropharyngealem Training von 14 auf 8. Auch der Schweregrad des OSAS wurde vermindert: Der ApnoeHypopnoe-Index (AHI) fiel von 22,4 auf 13,7 Ereignisse pro Stunde.

Interessanterweise nahm auch der Halsumfang von 39,6 auf $38,5 \mathrm{~cm}$ ab. Die tiefste Sauerstoffsättigung stieg signifikant von 83 auf $85 \%$. Der Score des Pittsburgh-Schlafqualität-Fragebogens verbesserte sich von 10,2 auf 6,9 .

\section{Kommentar}

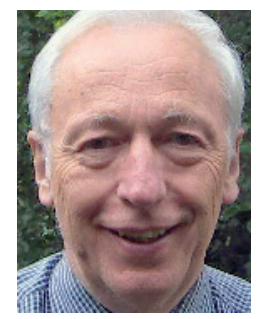

Prof. Dr. med. Karl-Heinz Rühle, Hagen

Nachdem mehrere Studien Hinweise darauf erbrachten, dass ein Training der oropharyngealen Muskulatur bei OSAS sinnvoll

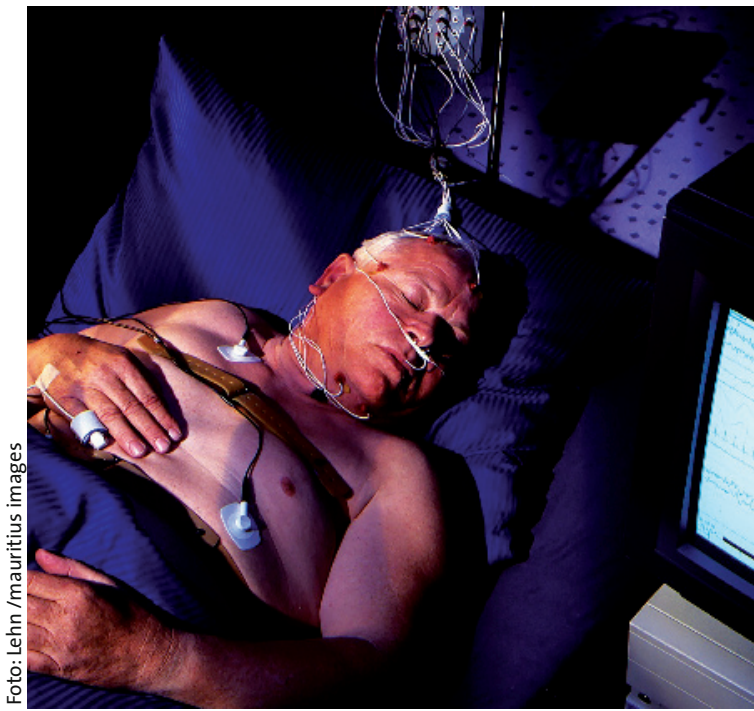

Im Schlaflabor wird der Effekt des Muskeltrainings überprüft.

sein kann, handelt es sich hier um die erste kontrollierte Studie, die einen relevanten Effekt nachwies. Die Reduktion des AHI um etwa 40\% liegt in derselben Größenordnung, die auch durch Protrusionsschienen erzielt wird. Besonders wichtig erscheint, dass der ESS-Score mit sechs Einheiten relevant zurückging.

Bei den Übungen handelt es sich um einen integrativen Ansatz, sodass der Effekt der einzelnen Komponenten nicht beurteilt werden kann. Sie stammen aus dem Bereich der Sprach- und Schlucktherapie. Der Effekt der einzelnen Übungen ist nicht evidenzbasiert. Die Übungen sind insgesamt sehr aufwendig und müssen regelmäßig durchgeführt werden.

Der Erfolg dürfte auch von der Erfahrung des Sprachtherapeuten abhängen. Außerdem wurde die Studie lediglich bei mittelgradigem OSAS durchgeführt. Die Methode sollte deshalb nicht bei schwergradigem OSAS eingesetzt werden.

Es muss bezweifelt werden, ob eine tägliche Übungsphase von 30 Minuten von den Patienten eingehalten wird. Deshalb ist es wahrscheinlich, dass sich diese Methode nur bei einer bestimmten Untergruppe von hoch motivierten Patienten mit OSAS durchsetzen wird. Wenn es allerdings gelänge, ein kürzeres Trainingsprogramm (5-10 Minuten pro Tag) mit den relevanten Übungen zu entwickeln, wäre dies ein wesentlicher Fortschritt zumindest für Patienten mit leichtem bis mittelgradigem OSAS. 